[This is the penultimate draft of a paper which appeared in the Journal of Medical Ethics 40/11 (2014): 774778: http://jme.bmj.com/content/early/2014/05/16/medethics-2013-101470?papetoc]

\title{
Preimplantation genetic diagnosis and rational choice under risk or uncertainty
}

Tomasz Żuradzki

\section{ABSTRACT}

In this paper I present an argument in favour of a parental duty to use preimplantation genetic diagnosis (PGD). I argue that if embryos created in vitro were able to decide for themselves in a rational manner, they would sometimes choose PGD as a method of selection. Couples, therefore, should respect their hypothetical choices on a principle similar to that of patient autonomy. My thesis shows that no matter which moral doctrine couples subscribe to, they ought to conduct the PGD procedure in the situations when it is impossible to implant all of the created embryos and if there is a significant risk for giving birth to a child with a serious condition. ${ }^{1}$

The article presents an argument supporting the claim that preimplantation genetic diagnosis (PGD) would be chosen as the method of selection by rational agents under some circumstances. The argumentation suggests that performing PGD is demanded from the perspective of embryos in the situations when not all of the created embryos can be implanted and there is a significant risk of passing on a serious condition. Therefore it is obligatory for couples because of a principle similar to the one concerning respect for patient autonomy. The argument can be accepted by advocates of various moral doctrines as it does not refer to any of them, but it is based on criteria of rational decisions in situations of risk or uncertainty and the principle of respect for patient autonomy. It also has an important advantage over standard arguments in support of PGD: it circumvents the non-

\footnotetext{
${ }^{1}$ An earlier version of the paper was presented at the 27th European Conference on Philosophy of Medicine and Health Care in Basel.
} 
identity problem, because it does not refer to benefits and harms for future people, but to hypothetical preferences of the interested parties.

The paper operates on the assumption-which does not seem to be controversialthat the decision about the choice of selection method is made in a society in which the in vitro fertilisation (IVF) procedure, and the production of so called 'surplus embryos', is allowed by law and practically performed. This assumption is not controversial because the procedure is permissible in all the European Union countries, including those where other issues related to the protection of the embryo and fetus are regulated in quite a restrictive manner (such as Ireland and Poland). It means that many couples who decide to have IVF are confronted with a dilemma: either they can let the clinician choose the best embryos for implantation by using some criteria such as PGD or they can choose randomly.

I will begin the paper with a hypothetical example which will be the basis of my further investigations (for the first time I have presented it in discussions of the Polish Bioethical Society 12 ) and then I will explain why it poses a dilemma similar to the decision problem during the IVF procedure. I will clarify some philosophical issues related to my interpretation of the example, in particular my use of the principle of patient autonomy and my understanding of the requirements of rationality. Then I will discuss possible objections to the argumentation and finally I will analyse whether we should genetically test for correlates of well-being.

\section{AN EXAMPLE}

Let us imagine that Anne and John, middle-aged siblings who undoubtedly enjoy full moral status according to every normative moral doctrine, have found themselves in an unfortunate situation which was not caused by either of them. They were kidnapped by a group of terrorists who are going to kill one of them soon. The terrorists are serious: their group is world famous for the regular kidnapping of pairs of tourists and then killing one of them and releasing the other. One of the kidnapped people has an as-yet undiagnosed genetic condition that can be easily tested for and will surely die within a year (or some other as-yet undiagnosed disease or impairment responsible for an early death). The other is perfectly healthy and one can reasonably expect that this person will live for 40 years more. The problem is that nobody knows which of them is healthy and which is ill. Luckily for them, it is possible to take a test which will unequivocally indicate the sick person. 
The terrorists are so vicious that they want the kidnapped themselves to decide in advance on the method of selection of the person to be killed: either they decide that the terrorists will kill the one who turns out to be sick after taking the test or they can rely on pure luck (if they do not make any decision themselves, the terrorists will decide randomly). In a hypothetical situation of choice presented in this example, we should assume that each rational agent would choose the test. On one hand, nobody suffers from the test: no matter if you flip a coin or take a test, the probability of surviving is identical, equalling 0.5 . On the other hand, if we assume that every rational agent wants to stay alive for a long time (in the frames of the present human scale and all other things being equal) and in the situation of risk he maximizes expected value, he should prefer to take the test. Let us assume that each year of life is a certain unit of measure. In cases when the choice was made by relying on pure luck, the expected value expressed in the years left to live amounts to 10.25 $\{0.5 \times[(40 \times 0.5)+(1 \times 0.5)]\}$; in the case when the choice was made on the basis of the test, it is $20\{0.5 \times 40\}$. It is thus because in the first case there is a 0.5 chance of being chosen and then a 0.5 probability that the chosen person is healthy and 0.5 that he or she is ill (therefore we have to add expected values that someone will live for the next 40 years and that someone will live for only 1 year). In the second case we know that the person chosen on the basis of the test will live for the next 40 years and the probability of being healthy (that means being chosen) is 0.5 .

This hypothetical example is analogical to a situation when there are two in vitro fertilised embryos and only one of them can be implanted: the kidnapped siblings are the embryos, the terrorists are the couple. The case is meant to represent many situations when embryos have conditions or impairments that are responsible for their premature death or inability to implant, for example, when one of the embryos' parents is a carrier of a disease that is inherited in an autosomal dominant manner. It means that the actual threat of an illness in such a case, in which there is a 0.5 probability of inheriting the illness for both embryos, is slightly different from the one described above, as the two randomly chosen embryos can be healthy or ill. However, this difference does not add anything significant to my argumentation: one can assume that in a situation when the siblings are simultaneously healthy or sick, the decision about staying alive is made randomly and such cases are omitted in this paper. To put it simply, I also assume-which is perhaps not far from the 
truth-that all of the human embryos remaining after in vitro fertilisation will be destroyed, sooner or later.

\section{THE ARGUMENT FROM PATIENT AUTONOMY}

This hypothetical example shows that in a particular situation of choice, when physicians are able to implant only some of the embryos, the decision to carry out PGD would be rationally preferred, by some observers and external decision-makers (because of reducing the costs of medical care, parental preferences, etc), and by the embryos themselves. So, couples should accept PGD as the desired method of selection out of a sense of respect for the hypothetical choices that would be made by their offspring.

Such an interpretation refers to the concept of autonomy: it is assumed that medical decisions should be made when respecting the autonomy of a patient, that is, making them a part of the decision making process related to them as well as allowing them to voice objections against the diagnosis, and healing and preventative treatments offered to them. The decisions are autonomous, when they are intentional, made with understanding and without any controlling influences, which is of course impossible when it comes to the case of embryos. If it is impossible to get to know the real will-which sometimes also happens in the case of adults-the surrogate should try to answer the question: "What would the patient choose to do if he could decide?". If the surrogate doesn't have sufficient knowledge to answer this question on the basis of the previous beliefs and choices of the patient, she is supposed to determine the answer by hypothetical choice. What would be the decision based on the hypothetical consent of the people from the example? In order to make this example closer to the decision making process in the case of embryo implantation, let us assume that Anne and John are unconscious, but the terrorists let an outsider made the decision on behalf of the kidnapped persons. This surrogate does not know the former views of the siblings and they have not made any advance directives.

The standard procedure in this kind of situation is clear: when making decisions on behalf of individuals whose particular preferences are unknown, a surrogate should be guided by the patient's best interests. In the classic formulation of Beauchamp and Childress it says that "a surrogate decision maker must determine the highest net benefit among the available options, assigning different weights to interests the patient has in each option and discounting or subtracting inherent risks or costs" ( p. 102).3 Since in the discussed case the 
risks are identical in every option (the 0.5 probability of death), a surrogate can concentrate only on the expected gains available for the kidnapped-assuming that one of them survives. To weigh up the options a surrogate must know the probabilities of different options and the expected good to be maximised. In the hypothetical example, the probabilities are known and I assumed that the years of life would be maximised-a fundamentally significant good when accomplishing any plan (additionally it is easily expressed in numbers). But, of course, one could argue that there are some other primary goods (the things which every rational agent is presumed to want as they are vital for all life plans4) that people would want to maximise, for example, some combination of expected years of life with their quality, for example, 'quality-adjusted life years' (QALY). Nothing in my argumentation prevents the consideration of other primary goods along with the expected years of life. Obviously, if the embryos were able to decide about themselves, the principle of patient autonomy would require asking them for agreement. In that casealthough I have stated above that the choice based on genetic testing would be preferred by every rational agent-it is not certain that actually every real agent, if found in the situation from the hypothetical example, would choose the maximisation of the expected years of life or other primary goods. In reality, the agents could apply their own particular criteria of decision making and such a decision should have to be respected. On a similar basis, physicians usually respect the decisions of patients who do not wish to undergo a treatment or procedure which would save their lives. Although there are extensive discussions as to whether a patient whose choices are clearly irrational can be treated as an autonomous agent, these discussions do not concern my usage of this principle. If the preferences or past choices of the patient are unknown, a surrogate is surely not permitted to assume that the patient would prefer a shorter life rather than a longer one or fewer primary goods than more.

The argument presented in this paper has been overlooked by contemporary bioethicists. A recent review article that identifies the main ethical and legal arguments in support of PDG names three types of ethical arguments.5 First, PGD is supposed to promote the well-being of future children; second, to broaden the spectrum of possibilities open to future children; third, to prevent future children from experiencing unfair disadvantages. The authors also mention other possible arguments, but finally do not treat them as genuine ones: that the use of PGD is related to some parental duties to promote the well-being of 
their descendants and that PGD would reduce the burden on society.

My argument has a few interesting advantages. One of them refers to the so-called nonidentity problem.6 A critic of arguments in favour of PGD might say that this selection method is neither beneficial nor harmful for those who will be born since, if the diagnosis was not carried out, different children could be born than those who will actually live (in terms of genetic material). However, this type of argumentation is not directed towards my point. I maintain that neither is taking the test beneficial for both siblings nor is it harmful that the decision is based on some random method. I only claim that such a decision should be made for the siblings from the examples, or the embryos in a given epistemic situation, when nobody knows (neither the interested parties themselves nor the agents carrying out the test) which agent is sick and which is healthy. I claim that this selection method should be chosen because it would be preferred by the interested parties themselves if they were guided by the maximisation of their expected life span.

Some proponents of PGD have defended their arguments from the non-identity objection by claiming that PGD increases the level of well-being not for individuals but for the class of children brought into being and that prospective parents should "aim to have the child who, given her genetic endowment, can be expected to enjoy most well-being in her life."7 This kind of argument refers to the view that PGD allows us to achieve various social or individual goals that in different contexts are treated as normal or even required, for example, the fulfilment of desires to have a healthy baby. The argument presented in this paper does not refer to any social or individual benefits and, at the same time, does not assume any kind of balancing of the interests of embryos with the potential social benefits or interests of other individuals (except for the cases discussed in the previous section where interests of embryos could be weighed against the interests of couples). Therefore it has a clear advantage: it could be accepted even by those who claim that early embryos have full moral status and their right to life can never be balanced with any other social or individual benefits.

\section{POSSIBLE OBJECTIONS}

One could argue that the example is not sufficiently analogous to in vitro procedures because during IVF usually more than two embryos are created and there can be different chances of the development of a genetic condition than in the main case. However, these 
factors would not change 'the mathematics' of my argument. Let us assume, for example, that four siblings have been captured by terrorists: Anne, Agnes, John and James. The chances that any of them have a condition similar to the one described in the main example is only 0.25 (the other circumstances are similar). In this situation it is also rational to conduct a test and decide on its basis. In cases when the choice was made randomly, the expected value expressed in the years of living amounts to 7.5625 $\{0.25 \times[(40 \times 0.75)+(1 \times 0.25)]\}$. There is the 0.25 probability of being chosen, the 0.75 probability that the chosen person is healthy (he or she will live for 40 years) and the 0.25 probability that the chosen person is ill (he or she will only live 1 year). In cases of testing there is an additional complication: since the test only shows which three out of the four are healthy, we have to decide which of those three will survive. I assume that the procedure has two stages: in the first we choose two healthy siblings on the basis of the test and, in the second, we decide which of them will survive by some random method. Therefore the expected value expressed in the years of living amounts to $10\{0.75 \times[40 \times 1 / 3]\}$. Other such modifications that would make the hypothetical examples closer to the reality of IVF procedures would also not change 'the mathematics' (eg, a different number of embryos, different chances of developing a condition, the implantation of more than one embryo, twin pregnancies, freezing of embryos and multiple rounds of PGD, taking into account other factors than genetic information that are relevant to a decision over which embryo to implant, etc).

Of course, one could argue that it is not always possible to know the precise probabilities of a genetic condition or life span as in my hypothetical examples. Therefore, the decisions are closer to those made under uncertainty, not under risk. Does ignorance concerning precise probabilities or about the nature of genetic illness influence the decision of people from my hypothetical examples? Certainly not, because if persons in the hypothetical situation only had an inkling that they might have a genetic disorder that would shorten their life, they have a reason to take this information into account. I assume that as long as they are rational they cannot choose to ignore this information (in reality, many people at risk of genetic diseases actually prefer not to be informed about their condition). Therefore, for any probability, no matter how small, that one of the siblings from the main example would have a genetic condition and would live a shorter life than the other, it is rational to decide on the basis of the test. On the other hand, when the probability of the 
genetic condition is small, the reasons to conduct genetic testing can be weak and, in the reality of IVF procedures, some other factors, for example, the risk related with the embryo biopsy procedure, can easily prevail. 8 This is the reason why I claim that couples have a duty to use PGD only if there is a significant risk of giving birth to a child with a serious genetic condition (even if they do not know the precise probabilities).

The risk related with the diagnosis generates an important complication for my argument. The current methods of diagnosis are not perfect: the embryo biopsy procedure during PGD involves a risk for embryos and the invasive prenatal diagnosis (PND) that is be recommended after PGD may lead to miscarriage. Some couples that desperately want to have a child may not want to accept these risks. They are willing to accept any child that is born, no matter whether it is disabled or not and they are able to give that child a relatively good life. There are two possible scenarios in these kinds of cases. In the first one, a couple does not go through IVF procedures or any type of PND, despite recommendations to do so and they try to conceive a child in the old-fashioned way, knowing that there is a significant risk that it will be born with a severe disability. The answer to the question of whether they are acting in a permissible way cannot be derived from my argumentation. I argued that couples are morally obliged to conduct PGD, only if they go through IVF, that is, when embryos have been already created and it is impossible to implant all of them. The second scenario is more relevant. In this case, a couple decides for IVF but they do not want to go through PGD and then PND, because they are afraid that either the embryos could die as a result of the diagnosis or that it would confirm a serious genetic condition. This case complicates my argumentation because it is a situation in which the interests of couples in having a child must be weighed against the interests of the embryos in maximizing their expected life span. The result of this weighing will depend on the value we attach to a couple's desire to have a child which is genetically related to them.

The most fundamental objection to my argument would try to investigate why a rational agent should maximise expected value in such situations of risk or uncertainty and why it is a requirement of rationality. The reason to prefer genetic tests is not that they increase the chances of survival, because an agent can be sure that no matter what she chooses (random choice or genetic testing) she will be dead in half (or, in the additional example at the beginning of this section, in three-quarters) of the results. The reason is that if an agent survives after the genetic testing, she can be sure that she is not worse off in 
terms of longevity than if she survives after the random choice and, on average according to the law of large numbers, she can rationally expect to be much better off. It would be easily visible if we imagined a larger population of kidnapped tourists: people in the population where agents decide to make genetic tests will live much longer than in a population in which people prefer random choice. Nevertheless, the longevity of a given population does not have to be the reason for individuals to carry on with the genetic test. I assume that the victims kidnapped by terrorists only want to live a long life and they are closer to fulfilling this aim if they choose the genetic test. This specific feature of my hypothetical situations (no matter which option an agent chooses, she is dead in half or more of the results) can help to rid us of the problem of risk aversion. In the standard situations of risk described in decision theory textbooks, an agent must decide, for example, if she prefers an option in which there is a 0.9 probability of earning $\$ 100$ (and a 0.1 probability of earning nothing) or an option with a 0.1 probability of earning $\$ 1000$ (and a 0.9 probability of earning nothing). The rationality of this choice depends on the circumstances (are you a millionaire or a starving poor man for whom $\$ 100$ is a fortune?), an agent's risk aversion (the preference for more certain results even if their expected value is lower) or the other preferences of an agent (eg, the scale of declining marginal utility). In contrast, in the examples I have discussed, these factors (and some others that can influence the choice under risk or uncertainty) do not play any important role. In particular, the risk aversion does not have any role to play, because the only parameter over which people can exercise the influence of their decisions is their own longevity-assuming they survive the test. Since they do not have any influence on the mere chances of survival, the risk attitude of agents does not matter (the same concerns the circumstances of the choice of the declining marginal utility of 1 year of life).

One could argue that discussions about the proper understating of the requirements of rationality might influence my argumentation. For example, one can ask whether I use an instrumental or substantive kind of rationality. In the hypothetical cases I have discussed, if we do not know the subjective wants of an agent, a surrogate should assume that an agent would rationally care about very specific things (ie, life expectancy). Therefore, it may seem that I do not limit my understanding of rationality to an account of the transmission of practical reasons (as a purely instrumental rationality would assume), but I also evaluate some of these agent preferences in terms of rationality. Thus it may seem that I use some 
substantive notion of rationality but, however, the preference for the longest possible life span can be understood in terms of the purely instrumental conception of rationality. It can be argued that being alive is a necessary means to achieve any end. So if I claim that every rational agent should prefer to live rather a longer life than a shorter one, it means that it is a requirement of instrumental rationality (Rawls argued that the same can be said about other primary goods because they are the inevitable means for all life plans4). Moreover, it is important to keep in mind that I use the term rationality in a very limited sense: only to evaluate the preferences of those agents whose real ones are unknown. I do not evaluate the preferences of real agents in terms of rationality. For example, the fact that it is required that couples maximize an embryo's life expectancy, does not mean that a person who knows he will definitely develop a genetic condition should rationally consider himself to be better off dead. To see this point, let us consider a modified example: Anna and John were kidnapped by a group of terrorists, who are going to kill one of them soon... John has a severe illness and doctors predict that he will only live for one more year. Anna is perfectly healthy and one can reasonably expect that she will live for 40 years more. In such a situation, the argument I defend does not claim that John ought to sacrifice himself for Anna. Of course, some people could argue that he ought to do it because of moral reasons. But in this case 'ought to' refers not to rational requirements or those of the principle of patient autonomy to which I refer. Therefore, this problem is beyond the scope of my argumentation.

\section{PGD AND WELL-BEING}

My thesis seems to be a bit weaker than that proposed recently by Mark Walker, who used 'a box game' to demonstrate that "If we are to best benefit potential supernumerary embryos, then we should genetically test for correlates of well-being."9 He presented the following thought-experiment:

Imagine you and I are sitting on identical very large boxes. One of us will win the contents of our box, the other will win nothing. We know that one box contains two Loonies (Canadian one-dollar coins) and the other contains $1,000,000$ Loonies. Neither of us knows who is sitting on the larger amount. The game show host, whose money it is, has said that he will 
use random selection to decide the winner, unless we both opt [...] to determine the winner by using a metal detector to select the box with the most coins. ( p. 217)

In the case of random selection, the expected pay-off is lower $(250000.50)$ than in the case of using a metal detector (500 000) (Walker mistakenly wrote 5000 000). It is thus-similarly to my case-because in the first case nobody knows if the randomly chosen box contains $\$ 1$ million or only $\$ 1$. Therefore there is a 0.5 probability of choosing the box on which you are sitting, and a 0.5 probability that the chosen box contains a $\$ 1$ million reward. In the second case (a metal detector), we know that the box chosen contains 1 million, and the probability that the box on which you are sitting will be chosen is 0.5 . Therefore the reason to prefer metal testing is not that it increases the odds of winning, but that when one of us wins, we should expect to win on average more than with random selection. Walker also adds that the loser is killed after the contest.

First of all it is true that, in an epistemic situation of either 'a box game' or the hypothetical examples I have discussed, the expected pay-off (either financial or expressed in the expected years of life) is higher when participants choose the equivalent of PGD. Therefore Walker is right to conclude that "metal detecting is the most rational method to use". But his thesis seems to be mistaken in other respects and his example is much less analogous to the reality of IVF procedures than mine. These kinds of lotteries cannot "best benefit supernumerary embryos", as he claims. It is so, because no matter which selection method we use, the same number of embryos is always implanted (or the same number of people survive). No matter who wins, all the benefits (either a higher pay-off or a longer life in my cases) go solely to the winners. Those who are not chosen using random methods or on the basis of the test do not benefit from the selection method as losers always have the same fate: death. So Walker uses the term 'benefit' in a very unusual sense: he suggests that every embryo (also supernumerary) will gain something because of PGD although only the winner will rake in the whole pot. He conflates the real and certain benefits of the winners with merely expected benefits, since he understands 'to benefit someone' is the equivalent of 'to give someone the same chance of survival, but with the higher expected pay-off'.

My thesis is more reliable because of two aspects. First, I do not claim that taking a test in my hypothetical example (or PGD procedure) is the best benefit for both sides separately. I only claim that this is what would be chosen by rational agents in particular 
situations of choice. And this is a reason why we have a moral duty to take PGD: we should respect the hypothetical choices of rational agents.

Second, I do not claim that genetic tests should be taken in order to find genetic correlates of well-being. This obviously leads to very interesting questions in the context of my example. If the difference between the siblings were based on the fact that one of them, as an effect of some factors present in the moment of decision-making, but unknown to anyone, will be healthy after being released by the terrorists and the other one will be sick (with a similar expected life span)? Or one of them will be more intelligent and the other less intelligent (assuming that the higher level of intelligence correlates with well-being). Or what if one of them had a character that lets one enjoy life more than the other? When it comes to all these cases, Walker claims that if it was only possible to test these features at the moment of decision-making, the decision about the one who has to survive should be made on the basis of a particular test (the most intelligent ones survive, those with the most optimistic personality, etc). Thus he argues that a procedure sometimes defined as 'positive eugenics' is beneficial for supernumerary embryos.

At this point I do not want to judge if his arguments are valid or not, but one should notice that the different thought experiment I have used implies that none of these stems from my argumentation. A rational agent has reasons to act on the basis of the expected life span, because this 'parameter' is certainly one of the primary goods which are necessary for every life plan. It is a similar case when it comes to health-at least in the case of very serious illnesses causing the permanent disability of some functions of an organism. However, neither the level of intelligence (except very low levels that can be treated as a disability) nor the predispositions of a character can be counted among those goods. Why? First of all, well-being is not a one dimensional property and cannot be 'cashed' in the same manner as the money reward in the box game. There are many reasons for this. One is that the well-being of individuals has many dimensions and depends on many different factors (especially their social environment) that are impossible to predict from their genetic endowment. Second, the way of understanding well-being itself is an important part of the moral doctrine or world view one accepts. So the advocates of various moral doctrines could understand quite different things under the label of 'well-being'.

It is, however, important to emphasise that my argumentation does not undermine the aim of PGD proposed by Walker (one should maximise the expected well-being). In this 
paper I have only proposed a new thought experiment and method of selection that would be preferred by every rational agent, irrespective of their worldview or the moral doctrine they believe in. Walker's criterion does not have this feature.

\section{CONCLUSION}

If PGD would be rationally preferred by the interested parties themselves, it has an important implication. It would mean that no matter which moral doctrine couples subscribe to, if they do not conduct the PGD procedure in the situation when it is impossible to implant all of the created embryos and when there is a significant risk of passing on a serious condition, they would not show proper respect for the hypothetical choices of their offspring. My argument demonstrates that even if somebody perceives the freezing and subsequent destruction of the surplus embryos remaining after an in vitro procedure to be the moral equivalent of killing adults, and thus sees potential parents who decide on PGD as just as immoral as the terrorists in the above example, she should still accept PGD as a method of embryo selection.

\section{FUNDING}

The research was supported by a grant 'luventus Plus' funded by the Polish Ministry of Science and Higher Education (no. IP2011065171).

\section{REFERENCES}

1 Żuradzki T. Niepewność na temat moralnego statusu embrionów ludzkich a preimplantacyjna diagnostyka genetyczna. Diametros 2012;34:179-89.

2 Żuradzki T. Ślepy traf a preimplantacyjna diagnostyka genetyczna. Przegląd Filozoficzny. Nowa Seria 2013;85(1):31-46.

3 Beauchamp TL, Childress JF. The principles of biomedical ethics. Oxford: Oxford University Press, 2001.

4 Rawls J. A theory of justice. Rev edn. Oxford: Oxford University Press, 1999.

5 Malek J, Daar J. The case for a parental duty to use preimplantation genetic diagnosis for medical Benefit. Am J Bioeth 2012;12(4):3-11. 
6 Parfit D. Reasons and persons. Oxford: Oxford University Press, 1984.

7 Savulescu J, Kahane G. The moral obligation to create children with the best chance of the best life. Bioethics 2009;23(5):274-90.

8 LaBonte ML. An analysis of US fertility centre educational materials suggests that informed consent for preimplantation genetic diagnosis may be inadequate. J Med Ethics 2012;38(8):479-84.

9 Walker M. Eugenic selection benefits embryos. Bioethics 2014;28(5):214-24. 